\title{
Segmental dynamics in poly(vinylidene fluoride) studied by dielectric, mechanical and nuclear magnetic resonance spectroscopies
}

\author{
Joanna Kaszyńska • Bożena Hilczer • Piotr Biskupski
}

Received: 1 June 2011 / Revised: 18 October 2011/Accepted: 31 October 2011 /

Published online: 10 November 2011

(C) The Author(s) 2011. This article is published with open access at Springerlink.com

\begin{abstract}
The dynamics of segmental motions in semicrystalline poly(vinylidene fluoride) has been studied by means of dielectric and mechanical spectroscopies and nuclear magnetic resonance method. The relaxation data, obtained from different techniques, over a wide temperature and frequency range, have been analyzed in terms of main-chain segmental motion, described by phenomenological HavriliakNegami function. The results indicate that the correlations between local conformational transitions in the amorphous phase are intermediate. Good agreement between the experimental and calculated data offers a contribution to the understanding of molecular dynamics in the glassy state of the polymer.
\end{abstract}

Keywords Polyvinylidene fluoride - Molecular dynamics $\cdot \alpha$-Relaxation · Mechanical and dielectric relaxation - Nuclear magnetic resonance

\section{Introduction}

The dynamics of molecular motions in systems which do not crystallize, even on slow cooling, but freeze into a glassy state is as yet not fully understood though various attempts have been made [1-6]. Glass-forming liquids and polymers are considered to be complex molecular systems and the structural $\alpha$-relaxation can not be described by Debye relaxation function with a single relaxation time. In order to get an unambiguous quantitative description of the molecular dynamics in these

\footnotetext{
J. Kaszyńska $(\bowtie) \cdot$ B. Hilczer

Institute of Molecular Physics, Polish Academy of Sciences, M. Smoluchowskiego 17, 60-179 Poznan, Poland

e-mail: bje@ifmpan.poznan.pl

P. Biskupski

Department of Physics, Adam Mickiewicz University, Umultowska 85, 61-614 Poznan, Poland
} 
complex systems, variable temperature and multifrequency study should be performed. It is obvious that there is no single spectroscopy or other technique that can cover the whole dynamical window, which ranges from thousandths of hertz up to thousand of gigahertz on the frequency scale; therefore, several methods have to be used and combined to study the dynamic phenomena. Among the methods to be used there are the quasielastic neutron scattering [6], the mechanical and dielectric relaxation [7-20], the rheo-dielectric method [21, 22], the nuclear magnetic resonance (NMR) [9, 23-27] and others. The relaxation phenomena observed by various techniques are expected to be different projection of the same structural relaxation mechanism. Furthermore, it should be possible to analyze the dynamical data, obtained by different methods, using the same model of motion $[6,28]$.

The subject of our paper is the poly(vinylidene fluoride) (PVDF) polymer. The PVDF and its copolymers with trifluoroethylene are important for applications in electromechanical and pyroelectric devices [29], in biomedical engineering [30] and recently also in high-density non-volatile memory cells of low writing voltage [31, 32].

PVDF is a semicrystalline polymer presenting pronounced polymorphic crystalline forms. It can crystallize into five possible conformations, three of which are most frequently occurring [33]. Almost planar zig-zag all-trans (TTTT) chain conformation of PVDF, packed in an orthorhombic unit cell exhibits ferroelectric properties-polymorph $\mathrm{I}(\beta)$. The $\mathrm{CF}_{2}$ dipoles are parallel to the orthorhombic $b$-axis and the long-range interactions are so strong that the Curie temperature is located close below the melting point. Non-polar polymorph II $(\alpha)$ consists of transgauche $\left(\mathrm{TGTG}^{\prime}\right)$ conformation of chains packed antiparallel in a monoclinic unit cell, whereas in the crystal form III $(\gamma)$ of PVDF the chains with TTTGTTTG' conformation are comprised in an orthorhombic polar unit cell. The conditions under which a specific conformation can be obtained depend strongly on the processing, thermal or mechanical treatments that the polymer undergoes. The ferroelectric-paraelectric phase transition is induced by cooperative modification in the chain conformation from all-trans to $\mathrm{TGTG}^{\prime}$ and TTTGTTTG'.

Despite many experimental and theoretical studies the glass transition still appears an unresolved problem in condensed matter physics. To describe nonDebye character of the $\alpha$-relaxation at temperatures above the glass transition, the main-chain segmental motion has been accounted for, by employing HavriliakNegami (HN) empirical equation, where both a distribution of correlation times in the system as well as the correlation of motion $[34,35]$ is assumed. By using HN formula, both the dielectric and the NMR experiments can be satisfactorily interpreted. The characteristic relaxation time $\tau(T)$, obtained from different methods, exhibits non-Arrhenius temperature behaviour, which has later been parameterized by Vogel-Tamman-Fulcher law [36-38] or equivalent WilliamsLandel-Ferry equation [7].

The main goal of this work was to examine the molecular dynamics of the PVDF polymer by means of NMR, dielectric and mechanical relaxation. The characterization yields experimental data covering the correlation time widow of ten decades and therefore allows to avoid erroneous dynamic information. 


\section{Experimental section}

\section{Samples}

PVDF powder from Nitrogenous Concern, Tarnów, Poland was used in NMR experiments, whereas the dielectric and mechanical spectroscopy studies were performed with radially oriented PVDF film. The film samples, $\sim 100 \mu \mathrm{m}$ thick and $11 \mathrm{~mm}$ in diameter, were obtained from PVDF powder by hot-pressing at $450 \mathrm{~K}$ and $600 \mathrm{MPa}$ for $30 \mathrm{~min}$. After hot-pressing the samples were cooled down to room temperature during $\sim 30$ min under the pressure and relaxed at room temperature without pressure for 3-5 days.

To characterize studied materials Differential Scanning Calotymetry (DSC), Wide Angle X-ray Scattering (WAXS) and NIR Raman spectroscopy were performed. DSC measurements were collected over a temperature range of 320-440 K on a Netzsch DSC 200 calorimeter with a scanning rate of 4 and $2 \mathrm{~K}$ per minute on heating and cooling, respectively. The sample, in the form of a powder, was placed in aluminium pans with pin hold lids. In the PVDF polymer thermogram (Fig. 1), single peak at $432.8 \mathrm{~K}$ related to the melting process is observed. Melting exotherm is shifted to lower temperatures at cooling process and located at $410 \mathrm{~K}$.

Wide angle X-ray scattering measurements of PVDF powder and films were performed with a PANalytical X'Pert PRO X-ray powder diffraction system and Cu $\mathrm{K}_{\alpha}$ radiation. The film was measured in a transmission mode with films placed between two Kapton foils. The measurements were done with $\mathrm{Cu} \mathrm{K}$ focusing X-ray mirror system $\left(\mathrm{Cu} \mathrm{K} \mathrm{K}_{\beta}\right.$ radiation was suppressed to $0.5 \%$ of its original level). The diffraction pattern was registered using the PIXcel detector. The data were collected for $2 \theta$ range from $10^{\circ}$ to $50^{\circ}$ with a $\Delta \theta$ step equal to $0.01^{\circ}$ during $16 \mathrm{~h}$ and worked out at the use of special computer program: X'Pert HighScor Plus version 2.0 (2004). Figure 2 shows an example of the X-ray pattern of hot-pressed PVDF film (the effect of Kapton films was extracted).

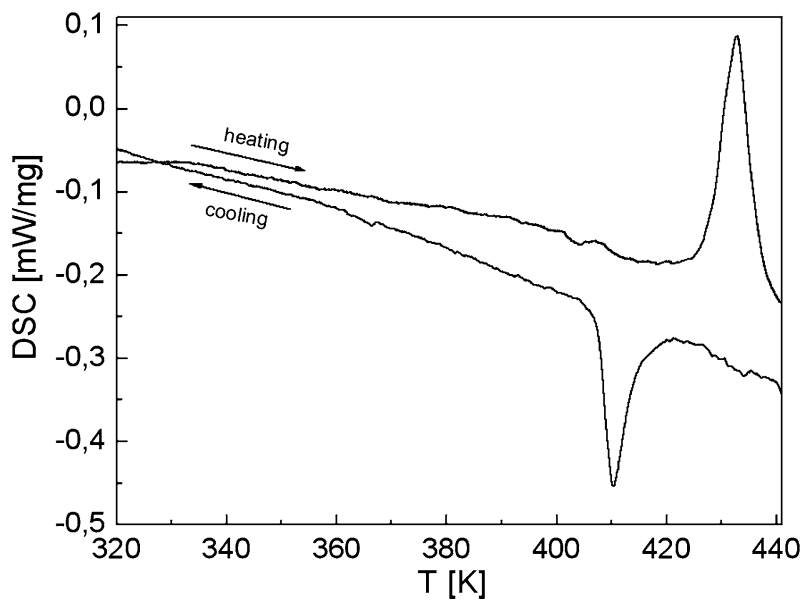

Fig. 1 DSC curve of the PVDF powder measured on heating and cooling the sample 


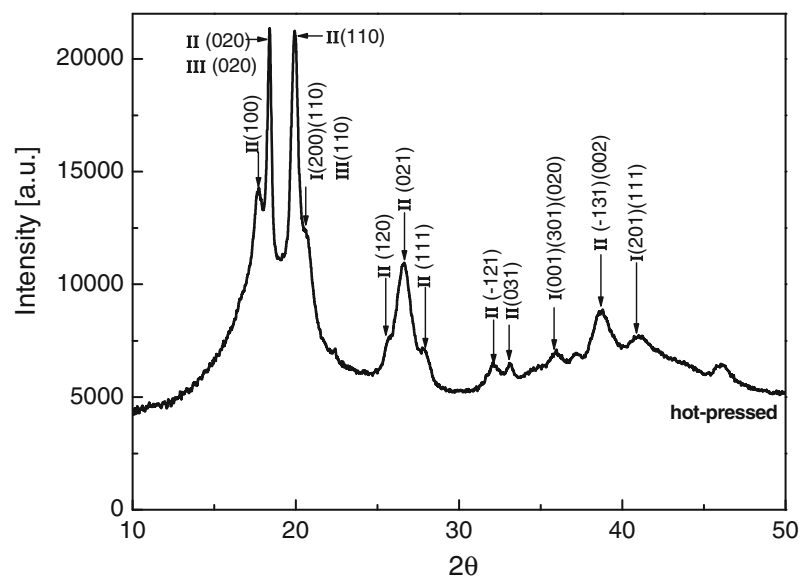

Fig. 2 Wide-angle X-ray diffraction profile of hot-pressed PVDF film

We found that the degree of crystallinity of hot-pressed film is $51 \%$ and that of the powder is $56 \%$. The amorphous phase of PVDF can be considered as consisting of statistical distribution of various rotational isomeric states.

To characterize local structure of the polymer at the molecular scale both in the crystalline and the amorphous phase NIR Raman spectroscopy was used. The spectra were recorded at room temperature using Bruker IFS 66FRA 106 spectrometer $\left(\lambda_{\text {inc }}=1064 \mathrm{~nm}, p=50 \mathrm{~mW}, \Delta_{\text {spectr }}=4 \mathrm{~cm}^{-1}\right)$. Figure 3 shows Raman spectra in the fingerprint wavenumber range for PVDF sample in the form of powder and the hot-pressed film. To characterize the phase content we used the assignment of Raman bands of PVDF published earlier [39] with the same notation: $v_{\mathrm{a}}$ and $v_{\mathrm{s}}$ asymmetric and symmetric stretching vibrations, respectively, $\delta$-bending

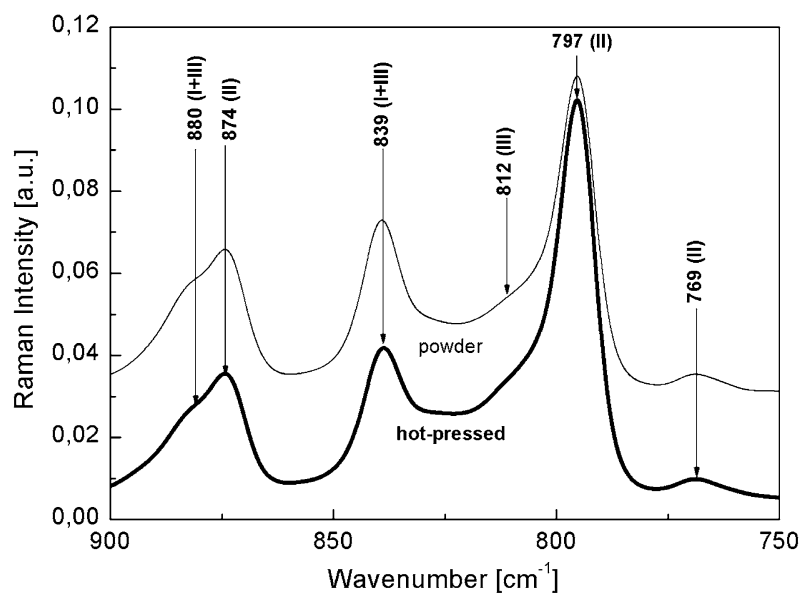

Fig. 3 Raman spectra in the fingerprint wavenumber range for PVDF sample in the form of powder (solid line) and hot-pressed film (thick line) 
vibration, $r$-rocking vibration. At high wavenumbers one observe two overlapping bands at $880 \mathrm{~cm}^{-1}$ originating from stretching vibrations $v_{\mathrm{s}}\left(\mathrm{CF}_{2}\right)+v(\mathrm{CC})$ in the phase I and phase III and at $874 \mathrm{~cm}^{-1}$ related to a combination of stretching and bending vibrations $v_{\mathrm{s}}(\mathrm{CC})+\delta(\mathrm{CCC})$ in the phase II. The intensity of the band, characteristic of the phases containing three and more $\mathrm{T}$ sequences, is lower than that of the TG conformation for both samples. A strong band at $839 \mathrm{~cm}^{-1}$, due to $r\left(\mathrm{CH}_{2}\right)+v_{\mathrm{a}}\left(\mathrm{CF}_{2}\right)$, is also present for the phase I and III whereas the $r\left(\mathrm{CH}_{2}\right)$ vibrations in the polar phase III are evident only as a shoulder. Very strong band at $797 \mathrm{~cm}^{-1}$ is characteristic of the $v_{\mathrm{s}}\left(\mathrm{CF}_{2}\right)$ vibrations in $\mathrm{TGTG}^{\prime}$ conformation. It appears that Raman spectra of PVDF in the form of powder and hot-pressed film do not differ considerably. The both samples contain all three polymorphous modifications and from the intensity ratio of the band at $797 \mathrm{~cm}^{-1}$ and the band at $839 \mathrm{~cm}^{-1}$ one can state that the hot-pressed sample is a little bit richer in the TG conformation than the PVDF powder.

\section{Dielectric measurements}

Dielectric response of PVDF films was measured in the frequency range $100 \mathrm{~Hz}-$ $1 \mathrm{MHz}$ using computer controlled HP-4284A LCR Meter. The samples with goldsputtered electrodes were placed in an Oxford Instruments Cryostat CF 1240, whereas the temperature was changed from 150 to $450 \mathrm{~K}$ at a rate of $1 \mathrm{~K} / \mathrm{min}$. The real and imaginary parts of the dielectric permittivity $\left(\varepsilon^{*}=\varepsilon^{\prime}-i \varepsilon^{\prime \prime}\right)$ were obtained as a function of frequency and temperature.

\section{Mechanical measurements}

The dynamic mechanical behaviour of PVDF rectangular hot-pressed films (3-5 mm $\times 5 \mathrm{~mm}$ ) was studied with NETZSCH DMA-242 using the tensile mode in frequency range 1-25 Hz. The dynamic force was $1 \mathrm{~N}$ and the proportion factor amounted to 1.2. The room temperature fatigue was assessed to be $\sim 5 \%$ after $4 \mathrm{~h}$. The experiments were performed in the temperature range between 150 and $470 \mathrm{~K}$ at a heating rate of $1 \mathrm{~K} / \mathrm{min}$ and temperature variation of real and imaginary components of the complex Young's modulus $\left(E^{*}=E^{\prime}-i E^{\prime \prime}\right)$ was obtained.

\section{Nuclear magnetic resonance}

For the NMR experiment, polymer powder was stored in glass tubes, evacuated at room temperature at $\sim 4 \times 10^{-6} \mathrm{hPa}$ for $20 \mathrm{~h}$ to remove oxygen, and sealed under vacuum. The proton spin-lattice relaxation $\left(T_{1}\right)$ measurements were carried out on a SXP 4/100 Bruker pulsed NMR spectrometer at a Larmor frequency of $90 \mathrm{MHz}$ between 200 and $440 \mathrm{~K}$. The temperature of the sample was controlled by means of a continuous nitrogen gas-flow system. An accuracy of the measured temperature was $1.0 \mathrm{~K}$. At least 30 -min time was allowed to stabilize the temperature of the samples. Spin-lattice relaxation time was determined by the inversion recovery method using a $180^{\circ}-\tau-90^{\circ}$ pulse sequence. The recovery of the magnetization was found to be exponential within experimental error at all temperatures. 


\section{Results and discussion}

\section{Dielectric relaxation}

The vacuum dipole moment of vinylidene fluoride unit $\mu_{(\mathrm{VDF})}=7.07 \times 10^{-30} \mathrm{Cm}$ originates from the distribution of positively charged protons and negatively charged fluorine ions and lies in the plane of $C-C$ bond (see Fig. 4). The dipoles are attached to the main chain allowing a study of the dielectric response related to segmental motions, local modes and reorientations leading from all-trans conformation in the crystalline state to TGTG' ${ }^{\prime}$ and TTTGTTTG ${ }^{\prime}$ conformations.

Normalized dielectric relaxation function can be expressed as:

$$
\frac{\varepsilon^{*}(\omega)-\varepsilon_{\infty}}{\varepsilon_{\mathrm{s}}-\varepsilon_{\infty}}=\Phi^{*}(\omega)
$$

where $\varepsilon_{\infty}$ and $\varepsilon_{\mathrm{s}}$ denote the non-relaxed and completely relaxed permittivity value, respectively.

The relaxation function $\Phi^{*}(\omega)$ of polymeric systems can be satisfactorily described by the Havriliak-Negami (HN) empirical equation [34, 35], which assumes a distribution of correlation times in the system and also the correlation of the motion, and is defined by:

$$
\Phi^{*}(\omega)=\frac{1}{\left[1+\left(\mathrm{i} \omega \tau_{\mathrm{HN}}\right)^{\alpha}\right]^{\beta}} .
$$

For the imaginary part of the permittivity it can be rewritten as:

$$
\begin{aligned}
\frac{\varepsilon^{\prime \prime}(\omega)}{\varepsilon_{\mathrm{S}}-\varepsilon_{\infty}}= & \sin \left[\beta \arctan \left(\frac{\sin \left(\frac{\alpha \pi}{2}\right)}{\left(\omega \tau_{\mathrm{HN}}\right)^{-\alpha}+\cos \left(\frac{\alpha \pi}{2}\right)}\right)\right] \\
& \times\left[1+2\left(\omega \tau_{\mathrm{HN}}\right)^{\alpha} \cos \left(\frac{\alpha \pi}{2}\right)+\left(\omega \tau_{\mathrm{HN}}\right)^{2 \alpha}\right]^{-\beta / 2},
\end{aligned}
$$

where $\alpha(0<\alpha \leq 1)$ and $\beta(0<\alpha \beta \leq 1)$ are two parameters characterizing the symmetric and asymmetric broadening of the dielectric band, respectively and $\tau_{\mathrm{HN}}$ is a characteristic time of the relaxation process. It should be noticed that the Eq. 1 leads to a simple Debye law for $\alpha=\beta=1$ and to the Cole-Cole [40] for $\beta=1$ and Davidson-Cole [41] function for $\alpha=1$.

Dielectric response in the low-temperature range, shown in Fig. 5a, is characteristic of segmental motion, i.e., freezing of dipolar motions in the amorphous phase of the semicrystalline polymer. The response is similar to that

Fig. 4 Schematic drawing of the spatial dipole moment arrangement of vinylidene fluoride (VDF) unit

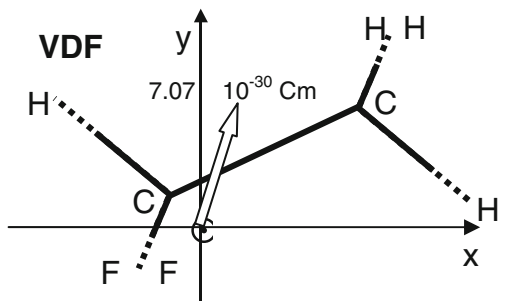



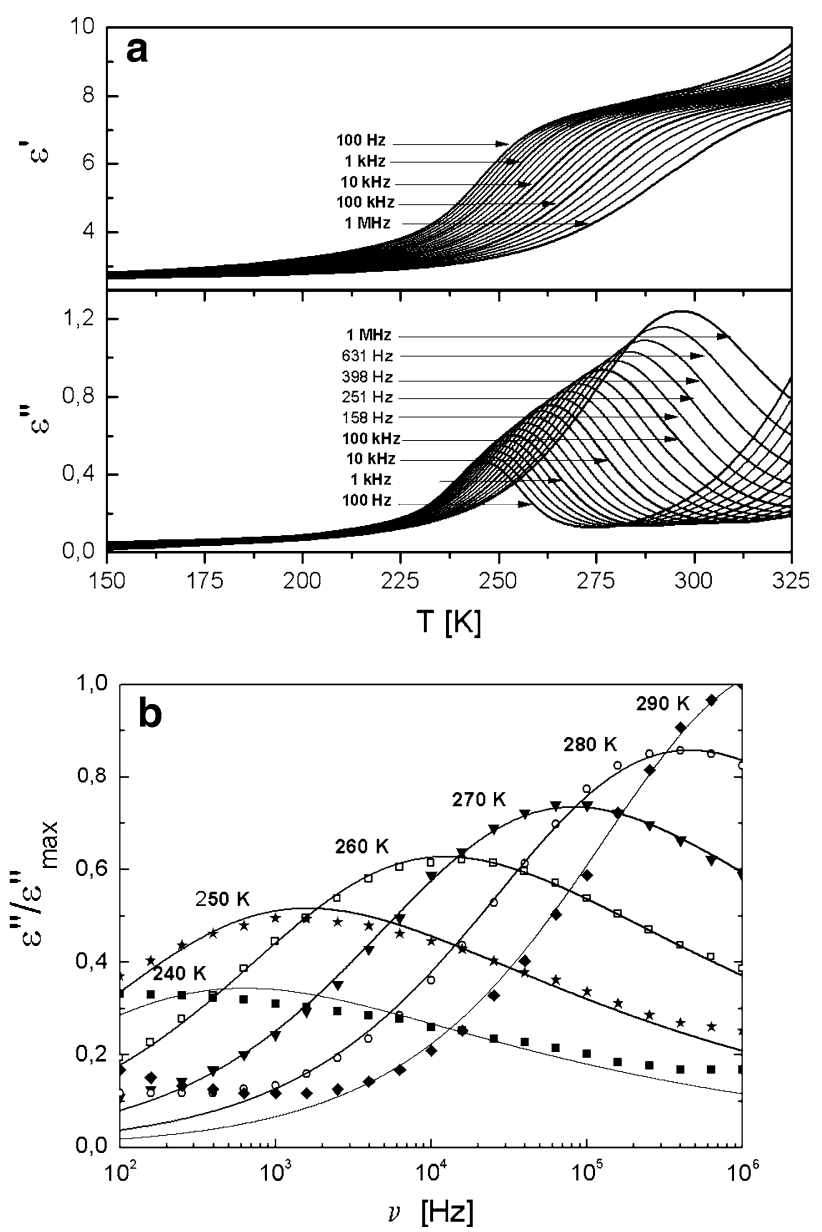

Fig. 5 a Temperature dependence of real and imaginary part of the dielectric permittivity of radially oriented PVDF at various frequencies. b Normalized imaginary part of the dielectric permittivity at 240 (filled square), 250 (star) 260 (open square), 270 (inverted triangle), 280 (open circle) and $290 \mathrm{~K}$ (diamond). The solid lines represent the HN fit with $\alpha=0.56$ and $\beta=0.36$

reported earlier for PVDF [10, 15]. The dielectric dispersion shows cusp-like temperature dependence, whereas the dielectric absorption increases with temperature and the maxima shift towards higher temperature with increasing frequency. The results obtained for normalized imaginary part of the permittivity $\varepsilon^{\prime \prime}(\omega) / \varepsilon^{\prime \prime}{ }_{\max }$ at different temperatures are shown in Fig. 5b. It is clearly visible only at $260 \mathrm{~K}$ the loss curve is well defined in the whole measuring frequency window. Therefore, the fitting procedure of Eqs. 1-3 was performed in the following way: first we have determined $\alpha$ and $\beta$ parameters at $T=260 \mathrm{~K}$ and than we have adopted the obtained values for the other temperatures. The solid lines in Fig. 5b show the best least-squares fit to $\mathrm{HN}$ equation with $\alpha=0.56$ and $\beta=0.36$. The agreement between experimental and calculated curves is satisfactory. The temperature 
behaviour of the dielectric relaxation time $\tau_{\mathrm{HN}}$ determined from the above fitting procedure will be discussed below and combined with that of the mechanical and NMR relaxation times, characteristic of the same process.

\section{Mechanical relaxation}

Figure 6 shows mechanical response of PVDF in the temperature range of the segmental motions. Similar temperature variation of piezoelectric and electrostrictive response as well as mechanical storage and loss moduli were reported in PVDF earlier [14, 19, 42, 43] but here we measured both mechanical and dielectric response using the same hot-pressed PVDF films with well-defined degree of crystallinity and polymorphs content.

The frequency range of our dynamic mechanical measurements in PVDF polymer was not broad enough to define the whole mechanical loss modulus curve. As the $E^{\prime \prime}(\omega, T)$ response determines the relaxation frequencies $\omega$ and the relaxation time $\tau_{\mathrm{HN}} \sim 1 / \omega$ we used the mechanical response in the temperature regime (Fig. 6) to calculate the characteristic time $\tau_{\mathrm{HN}}$ values from the maximum of the absorption curves $E^{\prime \prime}(T)$. It should also be noticed that in the glass transition range, the behaviour of the mechanical loss modulus $E^{\prime \prime}$ (Fig. 6) is similar to that of dielectric losses $\varepsilon^{\prime \prime}$ (Fig. 5a), i.e., they increase with increasing frequency.

\section{Nuclear magnetic resonance}

Proton spin-lattice relaxation time $T_{1}$ versus inverse of temperature for PVDF powder, plotted in a logarithmic scale, is shown in Fig. 7. The data display a single, quite broad and asymmetric minimum of $0.157 \mathrm{~s}$ at $329.9 \mathrm{~K}$, related to segmental motion in the amorphous phase. The uncertainty of the measurements is about $\pm 8 \%$ and is represented by the size of the symbols. Above $430 \mathrm{~K}$ a sudden decrease in

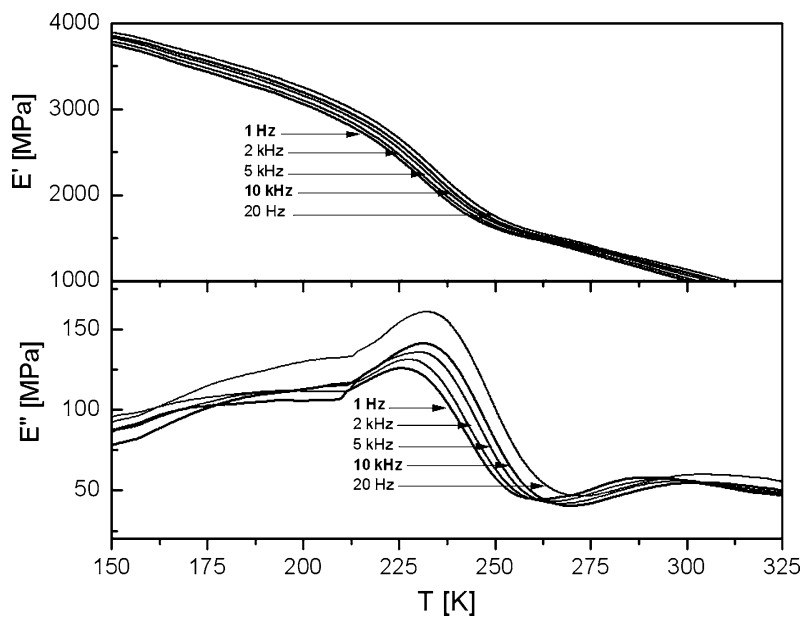

Fig. 6 Real and imaginary part of the complex dynamical modulus at various frequencies 


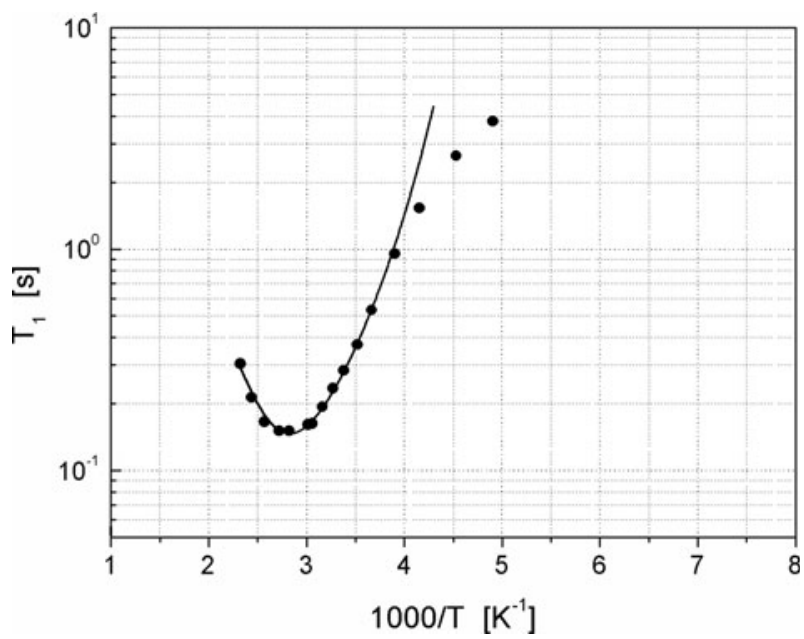

Fig. $7{ }^{1} \mathrm{H}$ spin-lattice relaxation time versus reciprocal temperature in PVDF polymer at the Larmor frequency of $90.00 \mathrm{MHz}$. Solid line represents the best theoretical fit of the HN function with $\alpha=0.59$ and $\beta=0.35$

$T_{1}$ vs $1000 / T$ was observed (not shown in Fig. 7). This change is attributed to the melting process in polymer and its value is in a good agreement with the corresponding melting temperature (see Fig. 1).

A qualitative analysis of the spin-lattice relaxation behaviour was based on the assumption that temperature dependence of $T_{1}$, as governed by the dipolar interaction modulated by the motional process, can be written as a linear combination of spectral densities $J\left(\omega_{\mathrm{L}}\right)$ [44]:

$$
\frac{1}{T_{1}}=C\left[J\left(\omega_{\mathrm{L}}\right)+4 J\left(2 \omega_{\mathrm{L}}\right)\right]
$$

where $\omega_{\mathrm{L}}$ denotes Larmor frequency and $C$ is a constant related to the fraction of the second moment, which corresponds to the dipolar interaction averaged by the motional process under consideration. The spectral density $J(\omega)$ can be expressed in terms of motional correlation times by means of equations depending on the theoretical or semiempirical model chosen to describe the molecular dynamics. In our case, the spectral density is based on Havriliak-Negami relaxation function [35] and is defined by [45]:

$$
\begin{aligned}
J(\omega)= & \frac{2}{\omega} \sin \left[\beta \arctan \left(\frac{\left(\omega \tau_{\mathrm{HN}}\right)^{\alpha} \sin \left(\frac{\alpha \pi}{2}\right)}{1+\left(\omega \tau_{\mathrm{HN}}\right)^{\alpha} \cos \left(\frac{\alpha \pi}{2}\right)}\right)\right] \\
& \times\left[1+\left(\omega \tau_{\mathrm{HN}}\right)^{2 \alpha}+2\left(\omega \tau_{\mathrm{HN}}\right)^{\alpha} \cos \left(\frac{\delta \pi}{2}\right)\right]^{-\beta / 2} .
\end{aligned}
$$

For the systems, which deviate most strongly from Arrhenius behaviour, the temperature dependence of a correlation time $\tau_{\mathrm{HN}}$ can be analyzed using the VogelTamman-Fulcher (VTF) formula [36-38]: 


$$
\tau_{\mathrm{HN}}=\tau_{0} \exp \left(\frac{B}{T-T_{\mathrm{VTF}}}\right),
$$

where $\tau_{0}$ is the relaxation time in the limit of high temperature, $B$ is related to the apparent activation energy $\left(B=\left[E_{\mathrm{a}}\left(1-T_{\mathrm{VTF}} / T\right)^{2}\right] / R\right)$, and $T_{\mathrm{VTF}}$ is the empirical VogelTamman-Fulcher temperature, which is usually $50 \mathrm{~K}$ lower than the glass transition temperature $T_{\mathrm{g}}$, below which the segmental motion of the main chain is completely absent. The dynamic glass transition temperature $\left(T_{\mathrm{g}}^{\mathrm{DRS}}\right)$ can be calculated [46]:

$$
T_{\mathrm{g}}^{\mathrm{DRS}}=T_{\text {ref }}=\frac{B}{\ln \left(1 / \tau_{0}\right)}+T_{\mathrm{VTF}}
$$

where $T_{\text {ref }}$ is defined as the temperature at which the segmental relaxation time is $1 \mathrm{~s}$ $\left(\tau_{\max }=1 \mathrm{~s}\right)$.

The fragility, $F$ which is a measure of the ability of a material to change its conformation across the glass transition region [47], can be calculated from the VTF fitting parameters using [48]:

$$
F=\frac{B / T_{\text {ref }}}{\ln (10)\left(1-T_{\mathrm{VTF}} / T_{\text {ref }}\right)^{2}}
$$

Larger fragility has been correlated with stronger intermolecular coupling or larger segmental size [48, 49].

Formalism mathematically equivalent to the VTF equation and also often used to characterize polymeric systems is the Williams-Landel-Ferry (WLF) equation [7]:

$$
\log a_{10}\left[\tau_{\mathrm{HN}}(T)\right]=\frac{C_{1}\left(T-T^{*}\right)}{\left[T-\left(T^{*}-C_{2}\right)\right]}
$$

where $C_{1}$ and $C_{2}$ are constants and $T^{*}$ is a reference temperature.

When the measurement range includes the glass transition temperature $T_{\mathrm{g}}$, obtained from calorimetry or dilatometry, it appears natural to choose $T_{\mathrm{g}}$ as $T^{*}$. If $T_{\mathrm{g}}$ was defined in a consistent way by measurement of thermal or volumetric changes at fixed scan rate $Q$, usually $1 \mathrm{~K} / \mathrm{min}$, then the parameters $C_{1}$ and $C_{2}$ appeared to have universal values of 17.4 and $51.6 \mathrm{~K}$, respectively [46]. $C_{1}$ and $C_{2}$ are related to the VTF parameters (if $T_{\mathrm{g}}$ is used as a reference temperature) through the relations $C_{2}=T_{\mathrm{g}}-T_{\mathrm{VTF}}$ and $C_{1}=B /\left(2.303 C_{2}\right)$.

To analyzed the NMR relaxation data Eq. 4 after inserting Eqs. 5 and 6 was used to fit the experimental data on Fig. 7, in the temperature range from 272 to $430 \mathrm{~K}$. The best fit was judged as that with the lowest $\chi^{2}$ value. The solid line in Fig. 7 represents the best result of this routine with $\alpha=0.59$ and $\beta=0.35$. These values are close to those obtained from the dielectric measurements above and the agreement between experimental and calculated curves is acceptable. The values of the characteristic time $\tau_{\mathrm{HN}}(T)$ in the temperature range covered experimentally can be determined for a given set of $\alpha$ and $\beta$ parameters, using Eqs. 4-6.

The temperature dependence of characteristic relaxation time $\tau_{\mathrm{HN}}$, obtained from dielectric and NMR spectroscopy experiments together with mechanical relaxation data is shown in Fig. 8. 


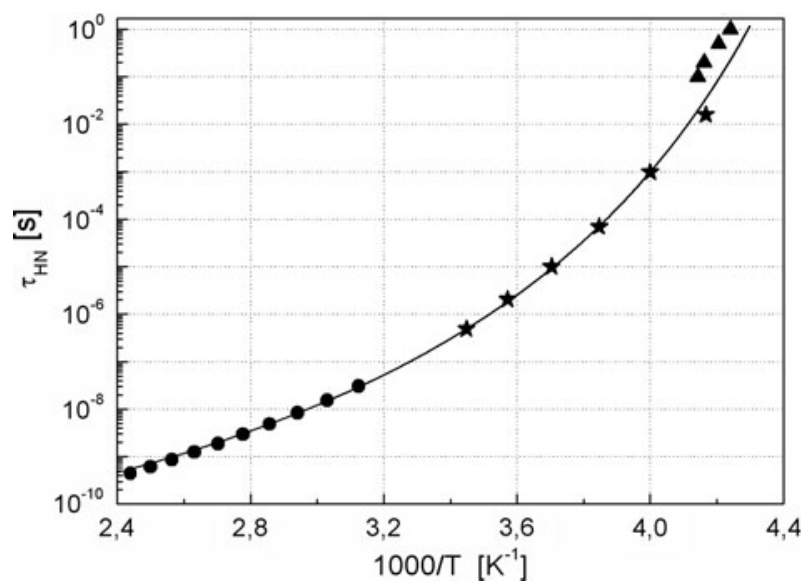

Fig. 8 Temperature dependence of the HN relaxation times for the $\alpha$-process of PVDF obtained by mechanical (triangle), dielectric (star) and NMR (circle) measurements. The solid line corresponds to the Vogel-Tamman-Fulcher fit defined by Eq. 6

Table 1 Vogel-Tamman-Fulcher parameters for the $\alpha$-relaxation process

\begin{tabular}{llllll}
\hline Sample & $\tau_{0}(s)$ & $B(\mathrm{~K})$ & $T_{\mathrm{VTF}}(\mathrm{K})$ & $T_{\mathrm{g}}^{\mathrm{DRS}}(\mathrm{K})^{\mathrm{a}}$ & $F^{\mathrm{b}}$ \\
\hline PVDF & $1.23 \times 10^{-12}$ & 1388 & 182 & 232 & 55 \\
\hline
\end{tabular}

${ }^{a}$ Defined by Eq. 7

b Calculated using Eq. 8

It is visible that the values of $\tau_{\mathrm{HN}}(T)$ obtained by different relaxation experimental techniques show the same, non-Arrhenius temperature behaviour and are of similar magnitude. Therefore, the temperature behaviour of the relaxation times from dielectric, mechanical and NMR can be parameterized by one VogelTamman-Fulcher law (Eq. 6). The fitted values of $\tau_{0}, B$ and $T_{\mathrm{VTF}}$ are listed in Table 1 along with the calculated $T_{\text {ref }}$ and $F$ values. As can be seen in the Fig. 8 (solid line), the VTF law fits very well the temperature dependence of the correlation time $\tau_{\mathrm{HN}}$ of the macroscopic $\alpha$-relaxation. The small deviation of the correlation times (triangles) obtained from the mechanical relaxation is due to the different method of the calculation $\tau_{\mathrm{HN}}$ in relation to those acquired from dielectric and nuclear magnetic relaxations.

As seen from Table 1 , the temperature $T_{\mathrm{VTF}}$ is $51 \mathrm{~K}$ lower than the glass transition temperature $T_{\mathrm{g}}$. In addition the calorimetrical $T_{\mathrm{g}}$ [50] and the calculated dynamic glass transition $T_{\mathrm{g}}^{\mathrm{DRS}}$ values are consistent.

From the equivalent Williams-Landel-Ferry equation (Eq. 9) we obtained $C_{1}=12.2$ and $C_{2}=56 \mathrm{~K}$. Though these values are not very adjacent to the so called "universal" ones $\left(C_{1}=17.5\right.$ and $C_{2}=52 \mathrm{~K}$, respectively) they are still, however, within the range of numbers commonly available for various polymers $[9,26]$.

Compatible values of $\alpha$ and $\beta$ parameters of the Havriliak-Negami equation have been determined from the fitting procedure of the dielectric $(\alpha=0.56, \beta=0.36)$ 
and NMR $(\alpha=0.59, \beta=0.35)$ data. These values indicate that the segmental main-chain motion are intermediate correlated $(\alpha \approx 0.57)$ and strongly distributed $(\alpha \beta \approx 0.21)$. The value of $C$ constant (Eq. 4) amounts to $1.86 \times 10^{9} \mathrm{~s}^{-2}$, and is of the expected order of magnitude.

\section{Conclusion}

We have shown that the combined dielectric, nuclear proton and mechanical relaxation measurements, performed over a wide temperature and frequency range on PVDF polymer, are a powerful tools for the characterization of molecular dynamics and the $\alpha$-relaxation in such complex systems.

Havriliak-Negami empirical equation was used to analyze the relaxation data and the obtained results show that the dynamics of segmental motion can be well described by the same $H N$ parameters $(\alpha \approx 0.57$ and $\beta \approx 0.35)$ in a wide time range. This indicates that the correlations between local conformational transitions in the amorphous phase of PVDF are intermediate and characterized by a pronounced distribution of the correlation times. As PVDF is a semicrystalline polymer it is not possible to determine directly "stretching" of the exponential behaviour, therefore we characterized the temperature dependence of the relaxation times. The temperature variation of the characteristic times $\tau_{\mathrm{HN}}$, deduced from our analysis, deviates from the Arrhenius behaviour and can be described by VogelTamman-Fulcher, or equivalent Williams-Landel-Ferry equations. The values of $\tau_{\mathrm{HN}}(T)$ determined by different relaxation experimental techniques consistently fit to Vogel-Tamman-Fulcher law with the same values of $\tau_{0}=1.23 \times 10^{-12} \mathrm{~s}$, $B=1388 \mathrm{~K}$ and $T_{\mathrm{VTF}}=182 \mathrm{~K}$ in the whole temperature range.

Acknowledgment This work is supported by the Grant No. N202 260534 from the Ministry of Science and Higher Education in Poland. The authors would like to thank A. Pietraszko from Institute of Low Temperature and Structural Research, PAS, Wrocław for WAXS measurements and M. Połomska from Institute of Molecular Physics, PAS, Poznań for Raman spectroscopy experiments.

Open Access This article is distributed under the terms of the Creative Commons Attribution Noncommercial License which permits any noncommercial use, distribution, and reproduction in any medium, provided the original author(s) and source are credited.

\section{References}

1. Donth E-J (2001) The glass transition. Relaxation dynamics in liquids and disordered materials. Springer Verlag GmbH, Berlin, Heidelberg, New York

2. Ngai KL (2000) Dynamic and thermodynamic properties of glass-forming substances. J Non-Cryst Solids 275:7-51

3. Ngai KL, Grzybowska K, Grzybowski A, Kamińska E, Kamiński K, Paluch M, Capaccioli S (2008) Recent advances in fundamental understanding of glass transition. J Non-Cryst Solids 354:5085-5088

4. Prevosto D, Capaccioli S, Luchesi M, Rolla PA, Ngai KL (2009) Does the entropy and volume dependence of the structural $\alpha$-relaxation originate from the Johari-Goldstein $\beta$-relaxation? J NonCryst Solids 355:705-711 
5. Jiang B, Kasapis S, Kontogiorgos V (2011) Combined use of the free volume and coupling theories in the glass transition of polysaccharide/co-solute systems. Carbohydr Polym 83:926-933

6. Colmenero J, Arbe A, Alegria A (1993) Crossover from Debye to non-Debye dynamical behavior of the $\alpha$ relaxation observed by quasielastic neutron scattering in a glass-forming polymer. Phys Rev Lett 71:2603-2606

7. Williams LM, Landel RF, Ferry JD (1955) The temperature dependence of relaxation mechanisms in amorphous polymers and other glass-forming liquids. J Am Chem Soc 77:3701-3707

8. Coburn JC, Boyd RH (1986) Dielectric relaxation in poly(ethylene terephthalate). Macromolecules 19:2238-2245

9. Colmenero J, Alegria A, Alberdi JM, Alvarez F, Fick B (1991) Dynamics of the $\alpha$ relaxation of a glass-forming polymeric system: dielectric, mechanical nuclear magnetic resonance, and neutron scattering studies. Phys Rev B 44:7321-7329

10. Mijovic J, Sy J-W, Kwei TK (1997) Reorientational dynamics of dipoles in poly(vinylidene fluoride)/ poly(methyl methacrylate) (PVDF/PMMA) blends by dielectric spectroscopy. Macromolecules 30:3042-3050

11. Sy JW, Mijovic J (2000) Reorientational dynamics of poly(vinylidene fluoride)/poly(methylmethacrylate) blends by broad-band dielectric relaxation spectroscopy. Macromolecules 33:933-946

12. Becker O, Simon GP, Rieckmann T, Forsythe J, Rosua R, Völker S, O’Shea M (2001) Dielectric relaxation spectroscopy of reactively blended amorphous poly(ethylene terephthalate)-poly(ethylene naphthalate) films. Polymer 42:1921-1929

13. Ghosh A, Naskar AK, Khastgir D, De SK (2001) Dielectric properties of blends of silicone rubber and tetrafluoroethylene/polypropylene/vinylidenefluoride terpolymer. Polymer 42:9849-9853

14. El Mohajir B-E, Heymans N (2001) Changes in structural and mechanical behaviour of PVDF with processing and thermomechanical treatments. 1. Change in structure. Polymer 42:5661-5667

15. Hilczer B, Kułek J, Markiewicz E, Kosec M, Malič B (2002) Dielectric relaxation in ferroelectric PZT-PVDF nanocomposites. J Non-Cryst Solids 305:167-173

16. Mierzwa M, Floudas G, Dorgan J, Knauss D, Wegner J (2002) Local and global dynamics of polylactides. A dielectric spectroscopy study. J Non-Cryst Solids 307-310:296-303

17. Roland CM, Casalini R (2003) Temperature and volume effects on local segmental relaxation in poly(vinyl acetate). Macromolecules 36:1361-1366

18. Robertson CG, Rademacher CM (2004) Coupling model interpretation of thermorheological complexity in polybutadienes with varied microstructure. Macromolecules 37:10009-10017

19. Mano JF, Sencadas V, Mello Costa A, Lanceros-Mendez S (2004) Dynamic mechanical analysis and creep behaviour of $\beta$-PVDF films. Mater Sci Eng A 370:336-340

20. Casalini R, Roland CM (2010) Effect of crosslinking on the secondary relaxation in polyvinylethylene. J Polym Sci Part B 48:582-587

21. Watanabe H, Matsumiya Y, Inoue TJ (2003) Rheo-dielectrics in oligomeric and polymeric: a review of recent findings. J Phys Condens Matter 15:S909-S921

22. Capaccioli S, Prevosto D, Best A, Hanewald A, Pakula T (2007) Application of the rheo-dielectric technique. J Non-Cryst Solids 353:4267-4272

23. McBrierty VJ, Douglass DC, Furukawa T (1982) Magnetic resonance and relaxation in a vinylidene fluoride/trifluoroethylene copolymer. Macromolecules 15:1063-1067

24. McBrierty VJ, Douglass DC, Furukawa T (1984) A nuclear magnetic resonance study of poled vinylidene fluoride/trifluoroethylene copolymer. Macromolecules 17:1136-1139

25. Pshorn U, Rossler E, Sillescu H, Kaufmann S, Schaefer D, Spiess HW (1991) Local and cooperative motions at the glass transition of polystyrene: information from one- and two-dimensional NMR as compared with other techniques. Macromolecules 24:398-402

26. Moe NE, Qiu XH, Ediger MD (2000) ${ }^{13}$ C NMR study of segmental dynamics of atactic polypropylene melts. Macromolecules 33:2145-2152

27. Forte C, Geppi M, Malvaldi M, Mattoli VJ (2004) Dynamics of an amorphous polymer by an improved NMR approach based on the simultaneous analysis of ${ }^{1} \mathrm{H}$ and ${ }^{13} \mathrm{C}$ relaxation times. J Phys Chem B 108:10832-10837

28. Roland CM (2010) Relaxation phenomena in vitrifying polymers and molecular liquids. Macromolecules 43:7875-7890

29. Uchino K (2000) Ferroelectric devices. Marcel Dekker., Inc., New York

30. Foster FS, Harasiewicz KA, Sherar MD (2000) A history of medical and biological imaging with polyvinylidene fluoride (PVDF) transducers. IEEE Trans Ultrason Ferroelectr Freq Control 47: 1363-1371 
31. Ducharme S, Gruverman A (2009) A simple nanoimprinting method creates arrays of ferroelectric polymer structures suitable for low-cost, non-volatile memories. Nat Mater 8:9-10

32. Hu Z, Tian M, Nysten B, Jonas AM (2009) Regular arrays of highly ordered ferroelectric polymer nanostructures for non-volatile low-voltage memories. Nat Mater 8:62-67

33. Tashiro K (1995) In: Nalwa HS (ed) Ferroelectric polymers. Marcel Dekker, New York, pp 63-181

34. Havriliak S, Negami SJ (1966) A complex plane analysis of $\alpha$-dispersions in some polymer systems. Polym Sci Part C: Polym Symp 14:99-117

35. Havriliak S, Negami S (1967) A complex plane representation of dielectric and mechanical relaxation processes in some polymers. Polymer 8:161-210

36. Vogel H (1921) Das Temperaturabhängigkeitgesetz der Viskosität von Flüssigkeiten. Physikalische Zeitschrift 22:645-646

37. Fulcher GS (1925) Analysis of recent measurements of the viscosity of glasses. J Am Ceram Soc 8:339-355

38. Tammann G, Hesse W (1926) Die Abhängigkeit der Viskosität von der Temperatur bei unterkühlten Flüssigkeiten. Zeitschrift für anorganische allgemeine Chemie 156:245-257

39. Kobayashi M, Tashiro K, Tadokoro H (1975) Molecular vibrations of three crystal forms of poly(vinylidene fluoride). Macromolecules 8:158-164

40. Cole KS, Cole RH (1941) Dispersion and absorption in dielectrics. 1. Alternating current characteristics. J Chem Phys 9:341-351

41. Davidson DW, Cole RH (1951) Dielectric relaxation in glycerol, propylene glycol, and n-propanol. J Chem Phys 19:1484-1490

42. Furukawa T, Aiba J, Fukada EJ (1979) Piezoelectric relaxation in polyvinylidene fluoride. Appl Phys 50:3615-3621

43. Linares A, Acosta JL (1997) Tensile and dynamic mechanical behaviour of polymer blends based on PVDF. Eur Polym J 33:467-473

44. McBrierty VJ, Packer KJ (1993) Nuclear magnetic in solid polymers. Cambridge University Press, Cambridge

45. Beckmann PA (1988) Spectral densities and nuclear spin relaxation in solids. Phys Rep 171:85-128

46. Santangelo PG, Roland CM (1998) Molecular weight dependence of fragility in polystyrene. Macromolecules 31:4581-4585

47. Angell CA (1991) Relaxation in liquids, polymers and plastic crystals-strong/fragile patterns and problems. J Non-Cryst Solids 13:131-133

48. Hodge IM (1996) Strong and fragile liquids-a brief critique. J Non-Cryst Solids 202:164-172

49. Erwin BM, Colby RH (2002) Temperature dependence of relaxation times and the length scale of cooperative motion for glass-forming liquids. J Non-Cryst Solids 307:225-231

50. Leonard C, Halary JL, Monnerie L, Micheron F (1984) DSC studies on the transition in poly(vinylidenefluoride) and some related copolymers. Polym Bull 11:195-202 